\title{
CLAUDIO SARTEA: LA BIOJURÍDICA ENTRE MEDICINA DEL DESEO Y EXTENSIÓN DEL DERECHO A LA INTIMIDAD
}

\section{THE BIOLAW BETWEEN MEDICINE OF WISHES AND EXTENSION OF THE RIGHT TO PRIVACY}

Resumen: La cultura moderna y postmoderna ha intentado reconstruir sobre fundamentos subjetivos todo el mundo humano, incluso el derecho y la medicina: hasta llegar a hacer coincidir la función de las respectivas profesiones con la satisfacción de las expectativas y de los deseos de los sujetos involucrados. Las paradojas y conflictos que van multiplicándose como consecuencia de dicho planteamiento sugieren retomarse en serio el bien objetivo salud (así como el bien justicia) en cuanto lugar común externo a los sujetos, que los junta al mismo tiempo que los separa e identifica.

\begin{abstract}
Modern and postmodern culture has attempted to reconstruct on subjective grounds all the human world, including law and medicine: up to match the role of both professions with the expectations and desires of the subjects involved. The paradoxes and the conflicts are multiplying as a result of this approach: that suggeststaking seriously again the objective concept of health (and justice), well as commonplace that joins and at the same time separates and identifies the persons.
\end{abstract}

Palabras claves: bioderecho; derecho a la intimidad; sujetivismo; orden.

Key Words: Biolaw; Right to Privacy; Subjectivism; Order.

\section{Premisa. Objetivo y sujetivo: en la medicina, en el derecho}

Sería dificil negar que, como a menudo repite Zygmunt Bauman ${ }^{1}$, nos encontramos hoy en tiemposlíquidos: líquida es la sociedad en que vivimos, líquidos los vínculos laborales y afectivos, líquidas las ideas, los partidos políticos, las relaciones internacionales, los compromisos éticos y religiosos. No se trata de una crítica ni de una constatación polémica, sino del intento de describir lo que son hoy en día las sociedades del bienestar occidentales, y la forma de vivir en ellas de las personas corrientes. Aunque no nos podamos contentar con describir las cosas, siempre una descripción adecuada constituye un buen punto de partida para reflexiones ulteriores.

\section{Una nueva medicina para una nueva salud}

La ciencia biomédica, como todo ámbito de actuación humana, sufre la misma liquidación: no tanto porque no vaya cada vez más afianzándose en su identitad científica y en el rigor de su método (aunque hasta en eso se puede notar alguna grieta ${ }^{2}$ ), sino

\footnotetext{
${ }^{1}$ Z. Bauman, Vida líquida, (2005), trad. A. Santos, Paidos, Barcelona, 2006; Amor líquido. Acerca de la fragilidad de los vínculos humanos, (2005), trad. M. Rosenberg y J. Arrambide, FCE, Madrid, 2006.

${ }^{2}$ Algún ejemplo, entre muchos: el Nobel chino del año pasado, la señora Youyou Tu, que ha cuidado la malaria con recursos de medicina tradicional china (la artemisia annua); el debate cada vez más veemente entre medicina oficial y medicinas alternativas (véase el interesante documento del Comité Nacional de Italia sobre este tema, de 2005: http://presidenza.governo.it/bioetica/testi/Medicine\%20Alternative.pdf);el reciente "caso Stamina", que ha llegado a los tribunales enfrentando dramáticamente pacientes oncológicos y médicos y envolucrando incluso el Ministerio de la Salud italiano. Un libro importante, al menos porque toma
} 
DOI: http://dx.doi.org/10.12795/IETSCIENTIA.2016.i02.11

porque su finalidad esencial y definitoria, la salud del ser humano, en que estriba su misma razón de ser así como la justificación social de su existencia ${ }^{3}$, va conociendo una importante evolucción semántica.

Todo conocimiento y toda práxis en medicina apuntansin duda, al fin y al cabo, a la salud de un individuo paciente: como infinitas veces se ha repetidodesde el juramento de Hipócrates hasta la Declaración Unesco de Bioética y Derechos Humanos ${ }^{4}$, art. 3: "1.Human dignity, human rights and fundamental freedoms are to be fully respected. 2.The interests and welfare of the individual should have priority over the sole interest of science or society". Entre otras cosas, esta regla aurea implica la prioridad normativa del individuo (por lo tanto, de su salud) sobre cualquier otro interés que la ciencia biomédica pueda legitimamente perseguir.

Lo que pasa en nuestros días es que el concepto mismo de salud se ha difuminado, o al menos se ha vuelto líquido de una manera hasta ahora desconocida. En falta de referencias definitivas, se tiende a consignar al paciente particular la evaluación de su estado y por consecuente el aprecio de la estrategia terapéutica a emprender con él: más aún, se construye la definición de salud y la calificación en términos de terapia de un tratamiento técnico, a partir de las expectativas y esperanzas del paciente mismo, y no desde la consideración objetiva de la eficacia en términos de curación de la enfermedad.

Un ejemplo puede aclarar bien esta afirmación: me refiero a las técnicas de reproducción humana artificial, cada vez más generalizadas y populares. En 2010, el doctor Edwards, que en Londres logró por primera vez, en 1978, el naciminento de un ser humano concebido fuera del cuerpo femenino, recibió el premio Nobel a la medicina. Es de interés volver a leer las motivaciones del premio ${ }^{5}$.

Today, IVF [In Vitro Fertilization] is an established therapy throughout the world. [...] IVF is a
safe and effective therapy. 20-30\% of fertilized eggs lead to the birth of a child.
Complications include premature births but are very rare, particularly when one egg only is
inserted into the mother. Long-term follow-up studies have shown that IVF children are as
healthy as other children. Approximately four million individuals have been born thanks to
IVF. Louise Brown and several other IVF children have given birth to children themselves;
this is probably the best evidence for the safety and success of IVF therapy. Today, Robert
Edwards' vision is a reality and brings joy to infertile people all over the world.

La "seguridad y la eficacia de la terapia", como se ve claramente en dichas afirmaciones, no quedan medidas por su objetiva actitud curativa (como se lee al final, las personas no salen de su condición de esterilidad sino permanecen en ella, así que si desean tener otro hijo deberán volver a las técnicas), sino por el logro del resultado final (niños sanos y capaces de reproducirse de forma natural), y en la "alegría" que ello en todo caso produce. Como es sabido, aunque en las palabras citadas todo esto no aparezca, la externalización de la singamia ha multiplicado las posibilidades reproductivas introduciendo problemas bioéticos y biojurídicos completamente inéditos, como él de la

en serio la cuestión teórica de la medicina y su problema epistemológico, es AA.VV., Filosofia della medicina, a cura di G. Federspil, P. Giaretta, C. Rugarli, C. Scandellari, P. Serra, Cortina, Milano, 2008.

${ }^{3}$ Es opinión del autor que la justificación social y por lo tanto jurídica de una profesión depende de su servicio a la comunidad en el lugar y en el tiempo dados: se permita referirse a C. Sartea, Deontologia. Filosofia del lavoro professionale, Giappichelli, Torino, 2010.

${ }^{4}$ http://portal.unesco.org/en/ev.php-URL_ID=31058\&URL_DO=DO_TOPIC\&URL_SECTION=201.html

5 "The 2010 Nobel Prize in Physiology or Medicine - Press Release". Nobelprize.org. Nobel Media AB 2014. Web. 21 Oct 2016. <http://www.nobelprize.org/nobel_prizes/medicine/laureates/2010/press.html> (son mías las cursivas). 
DOI: http://dx.doi.org/10.12795/IETSCIENTIA.2016.i02.11

fecundación heteróloga, él de la maternidad por surrogación, o las cuestiones dependientes de la crioconservación de embriones humanos, de su selección preimplantatoria, de su manipulación.

Más allá del ejemplo y de su carga problemática, no cabe duda de que la voluntad y los deseos y expectativas del paciente, así como su percepción de la situación, resultan esenciales, ya que al fin y al cabo siempre de su salud se trata. De todo esto se ocupan incluso los códigos deontológicos, que imponen a los médicos el deber de tener adecuadamente en cuenta los requerimientos y los deseos del enfermo - en conformidad, desde luego, con la Convención de Oviedo $^{6}$ de 1997 sobre Derechos Humanos y Biomedicina:"Article 9 - Previously expressed wishes. The previously expressed wishes relating to a medical intervention by a patient who is not, atthe time of the intervention, in a state to express his or her wishes shall be taken into account".Pero aquí la cuestión es muy distinta: aquí se hace coincidir el resultado clínico con la satisfacción de la expectativa del paciente y la realización de su petición.

Algo parecido pasa en la jurisprudencia sobre aborto, por lo menos en Italia. Como la ley de aborto en mi País (n. 194/1978) legaliza la interrupción del embarazo en los primeros noventa días, con tal que la mujer alegue motivaciones personales que se refieran de forma directa o indirecta a su salud física o psíquica, asistimos en las sentencias a la progresiva apertura a los estados psicológicos como motivo suficiente para exigir de forma plenamente legal la intervención cirúrgica ${ }^{7}$. Se trata del desarrollo de la idea clave con que elTribunal Constitucional italiano, ya en 1975, introdujo en el ordenamiento una excepción al principio general del respeto a la vida inocente admitiendo la supresión del feto "che persona deve ancora diventare", con tal que esté en juego la vida 0 aún solamente la salud de la madre, "che persona giá è"8. Aparece con claridad, también en casos como éstos, la sujeción de la misma calificación de la naturaleza de un acto profesional (delito punible o servicio debido, según los casos) a la mera voluntad de la "paciente". Aquí todo se muestra muy discutible, ya que resulta bastante arduo calificar de enfermedaden sentido técnico la situación de la mujer preña,y aún más arduo es sin duda calificar el desarrollo del embarazo de agresión - y seguramente no voluntaria - a su salud: cualquier sea el origen del embarazo ${ }^{9}$, su desarrollo fisiológico es independiente y no comporta de por sí daño a la vida de la mujer, menos que en casos muy excepcionales en los que no tendría mucho sentido hablar de aborto voluntario. En la

\footnotetext{
${ }^{6}$ https://rm.coe.int/CoERMPublicCommonSearchServices/DisplayDCTMContent?documentld=09000016800 $7 \mathrm{cf} 98$

7 Incluso hasta poner en tela de juicio la legitimidad de la objeción de conciencia, que se opondría al ejercicio del que cada vez más pretende presentarse como un derecho sujetivo de la mujer embarazada: véase en argumento el reciente consensus statement publicado en el blog de filosofía moral de la Oxford University (<http://blog.practicalethics.ox.ac.uk/2016/08/consensus-statement-on-conscientious-objection-inhealthcare/>), importante no por los argumentos, muy pobres y poco desarrollados, sino por el prestigio académico internacional de la institución y por la notoriedad de algunos de sus suscriptores.

${ }^{8}$ Corte Costituzionale italiana, sentenza n. 27 del 1975.

${ }^{9}$ En algunos ordenamientos el aborto ha sido legalizado a partir de casos de violación de la mujer (el caso más es el de Estados Unidos, que con la sentencia de 1973 Roe vs Wade permitió en todos los Estados la interrupción del embarazo - y se trataba de un caso de supuesta violación; pero no es una estrategia aislada, ya que por ejemplo en esos meses en Peru se discute la introducción del aborto legal para los casos de violación de la mujer). En nuestro razonamiento, lo que importa es subrayar que la calificación de la naturaleza propia de una actuación clínica no puede depender de las causas fácticas que han llevado a la "enfermedad" (cuya calificación tampoco depende de circustancias ocasionales) ni de la voluntad individual: el obrar del profesional responde a reglas y definiciones que necesariamente tienen cierta objetividad.
} 
cada vez más extensa jurisprudencia del "wrongful birth" y del "wrongful life", se juntan por lo tanto el subjetivismo médico y el subjetivismo jurídico: ya que la calificación del daño depende por completo de la subjetiva percepción de los interesados: desde el punto de vista del médico (en estos casos, del ginecólogo), su conducta podrá ser lícita o ilícita (y a la vez según las leges artis o en contra de ellas) en dependencia de la evaluación del sujeto asistido - lo cual no acaba de resultar paradójico además de fuente de graves incertidumbres jurídicas y existenciales ${ }^{10}$.

En otro distinto marco, se entiende solamente desde la misma perspectiva la idea de otra forma, incomprensible y, una vez más, paradójica - de que la muerte pueda constituir el objeto de un tratamiento médico, a través de la eutanasia y del suicidio asistido ${ }^{11}$. Teniendo en cuenta que la finalidad de la ciencia biomédica, si es que hay una, estriba en la salud del paciente, algo que niegue tan radicalmente esta finalidad como es dar la muerte al paciente mismo que la pida puede ser considerado el contenido específico de un acto médico solamente a condición de que la satisfacción de la voluntad del paciente, más que sus exigencias objetivas de salud psicofísica, constituya lo que la medicina persigue. Se impone, en otras palabras, un profundo cambio epistemológico en la misma autocomprension de la medicina, en orden a la actuación de los profesionales: hace falta que ellos lo sepan, se enteren y sobre todo lo acepten colectivamente, antes de considerar obvio que el cambio haya tenido lugar. La persistencia del fenómeno de la objeción de conciencia en los ámbitos cruciales del inicio y del final de la vida, y la reclamación del derecho a la objeción en los ordenamientos jurídicos en los que aborto, eutanasia y suicidio asistido resultan legales, son datos que deberían hacernos reflexionar con profundidad, evitando cuidadosamente - como personas racionales que todos queremos ser, sobre todo a la hora de decidir sobre la vida y la muerte de nuestros semejantes, y delante de su sufrimiento - los cortocircuitos del emotivismo y las ambigüedades de una pietas que más se parece a una forma de pietismo indebida, en que el paternalismo médico echado por la puerta vuelve a asomarse por la ventana ${ }^{12}$.Es como si todas las veces en que se intenta encontrar un lugar de contacto entre paciente y médico, distinto del "rational" clínico, algo esencial e irrinunciable queda perdido.

\section{Un nuevo derecho y los "nuevos derechos"}

Pasamos ahora a considerar la vertiente jurídica del asunto. Es muy llamativa la

\footnotetext{
${ }^{10}$ Para profundizar en un caso específico de jurisprudencia sobre "wrongful birth", se pide licencia para remitir a C. Sartea, "Bioetica giudiziaria in Italia: note critiche su una sentenza recente in tema di protezione della vita prenatale", en Ius Humani. Revista de Derecho, Vol. 4 (2014/2015), pp. 49-76.

11 Per un quadro aggiornato sulla situazione internazionale in materia eutanasia (legislazione ed applicazione delle regole permissive), cfr. E.J. Emanuel et alii, Attitudes and Practices of Euthanasia and Physician-Assisted Suicide in the United States, Canada, and Europe, JAMA, 2016; 316(1): 79-90.

12 Spagnolo habla de "suicidio assistito della autonomia": legalizar el suicidio asistido como se pretende ahora en Inglaterra implica el suicidio de la autonomía del paciente (ya que la petición de muerte corresponde casi siempre al resultado de una falta de objetividad de juicio: cfr., sobre este aspecto, la "postilla crítica" de Francesco D’Agostino al documento del Comité Nacional de Bioética de Italia "Rifiuto e rinuncia consapevole al trattamento sanitario nella relazione paziente-medico", del 24.X.2008, en <http://presidenza.governo.it/bioetica/pareri_abstract/rifiuto_rinuncia_consapevole_paziente_medico_24102 008.pdf>), y también el suicidio de la autonomía profesional y de la autonomía del razonamiento "quando, invece di formulare correttamente argomenti pro e contro, sulla cui soliditá o debolezza si basa il merito, si sostiene la non sinceritá degli argomenti degli oppositori, ritenendoli contaminati da un pregiudizio religioso" (A.G. Spagnolo, "Il suicidio assistito dell'autonomia", en Medicina e Morale, 2015/4, p. 535). Antes ya había hablado del derecho a la autodeterminación como de una entre las "paranoie della modernitá" F. D’Agostino, Introduzione ai lavori: paranoie della modernitá, en AA.VV., Autodeterminazione: un diritto di spessore costituzionale?, Giuffrè, Milano, 2010, p. 1.
} 
analogía: también en esta vertiente resulta patente el giro sujetivista que ha dado la perspectiva sobre el derecho, especialmente por lo que se refiere a los derechos humanos. Dichos derechos, planteados en términos de "metafísica de la sujetividad" (así Cotta $\left.{ }^{13}\right)$, se han vuelto en la pauta de las pretensiones arbitrarias e incontestables de los individuos: pero no solamente de las pretensiones fundamentadas en los vínculos sociales, según la perspectiva clásica, sino también de las que se derivan de deseos totalmente particulares, incluso los que son potencialmente conflictivos con esos mismos vínculos $^{14}$, como los que hemos encontrado más arriba.

Asistimos en otros términos a la juridificación de los sentimientos, a una forma inédita de "sentimentalismo jurídico": es decir a una relevancia completamente nueva de la esfera emocional del individuo en el discurso jurídico, que se manifiesta entre otras maneras en la transformación del deseo en derecho ${ }^{15}$. Ya no hace falta argumentar racionalmente para exigir la garantía ordinamental de una pretensión individual: en cuanto tal, es decir en cuanto promanante de la libertad de un individuo particular, y en ausencia de un prejuicio que pueda afectar a otros seres libres, es ésta la razón suficiente para lograr la plena protección de la ley o también, cuando una ley no exista, un reconocimiento jurisdiccional adecuado.

La fecundación artificial es un ejemplo en mi opinión muy claro, sobre todo en su vertiente heteróloga: es muy difícil afirmar que se trate de terapia ya que la pareja especialmente el miembro estéril de la pareja, cuyo gameto viene sustituido por el "donante" - no queda curada de su enfermedad: solamente se logra satisfacer el profundo deseo de genitorialidad con una ficción de descendencia y de pertenencia, conseguida a través de la entrega a la pareja estéril de un niño que pasará a ser "de" la misma pareja en virtud de una operación esencialmente lingüística y social, sin ningún fundamento biológico-genético ${ }^{16}$. Las semejanzas con la adopción vienen sistemáticamente difuminadas por quienes abogan en favor de la equiparación de las situaciones artificiales a las naturales: lo que pasa es que no es suficiente con cerrar los ojos para que no exista la luz del sol (aunque quien los cierre pueda luego afirmar que no la ve).

Otro ejemplo alumbrante es el del alquiler de útero: es bien sabido que en todo el

\footnotetext{
${ }^{13}$ S. Cotta, Attualitá e ambiguitá dei diritti fondamentali, in Id., I/ diritto come sistema di valori, San Paolo, Milano, 2004, pp. 9 y ss.

${ }^{14}$ Ya sea desde el punto de vista del paciente (no se olvide que según Aristóteles el suicidio quedaba prohibido no en fuerza del principio de indisponibilidad de la vida corporal, sino en fuerza de la justicia hacia la polis), ya sea desde el punto de vista del médico, vinculado al respeto de sus deberes deontológicos.

${ }^{15}$ Muy acertado por lo tanto resulta el título del último libro de F. Zanuso (ed.), Diritto e desiderio. Riflessioni biugiuridiche, Milano, FrancoAngeli, 2015, donde se intenta mostrar que si hace coincidir el derecho con la protección del deseo, un bioderecho es imposible, ya sea al comienzo de la vida, ya sea en su fase final.

${ }^{16}$ Se trata de un desplazamiento semántico que algunos ya consideran característico de nuestra época: véase el "Rapport Théry" (Paris, 2014), encargado por el gobierno francés a un grupo de expertos en ciencias sociales, en cuyas conclusiones claramente se afirma que "Lejos de excluir o ierarquizar, nosotros opinamos que el sentido de una reforma de la filiación debe ser el de hacer coexistir en igual dignidad tres modalidades para estabecerla: la generación por procreación física, la adopción, la generación por terceros donantes" (p. 18 del original francés, mia la traducción; visionado el 3 de noviembre de 2016 a 10.00 h.: $<$ http://www.justice.gouv.fr/include_htm/etat_des_savoirs/eds_thery-rapport-filiation-origines-parentalite2014.pdf>). Más compleja y problemática la perspectiva en la que se mueve una experta de psicología familiar como Silvia Vegetti Finzi (Oscurità dell'origine e bioetica della veritá, in AA.VV., Questioni di bioetica, a cura diS. Rodotà, Laterza, Roma-Bari, 1997). A quienes abogan en favor de la analogía entre adopción y fecundación artificial heteróloga (por terciedad de gametos o por alquiler de útero), habrá que aconsejar la importante lectura de F. D’Agostino, La figura paterna nella fecondazione assistita, en Id., Bioetica nella prospettiva della filosofia del diritto, Giappichelli, Torino, 1996, pp. 269 y ss.
} 
DOI: http://dx.doi.org/10.12795/IETSCIENTIA.2016.i02.11

mundo va montando la polémica entre los favorables a la práctica (en algunos casos, a condición de que se trate de surrogación oblativa y no comercial ${ }^{17}$ ), y los detractores: sobre todo las detractoras, ya que la batalla al útero alquilado se ha vuelto en una bandera del feminismo contemporáneo, al menos en parte decidido a impedir con todas sus fuerzas una nueva forma de explotación de la mujer ${ }^{18}$. A pesar de la general condena de la práctica de alquiler de útero, sobre todo en su vertiente lucrativa (por el riesgo de explotación ya señalado, más que por otras razones más enraigadas en la dignidad humana y en la protección de la genuina relación genitorial), van multiplicándose las señales de un callado pero cada vez más generalizado acostumbramiento: el reciente caso Paradiso y Campanelli, que involucra a una pareja italiana, es muy ilustrativo acerca del intento generalizado demostrar que no existiría diferencia jurídicamente relevante entre natural y artificial en lo que afecta a la generación humana. En este caso, y hasta la fecha, ya que pende recurso delante de la Grande Chambre, el Tribunal de Estrasburgo ha reconducido la situación al derecho a la intimidad del que habla el artículo 8 de la Convención Europea de Derechos Humanos ${ }^{19}$. Aunque de esta forma se evite entrar en el mérito de la cuestión, resulta patente que la orientación del Tribunal Europeo se dirige hacia la legitimación de otra práctica fundamentada en la ficción de genitorialidad, en este caso con referencia no a la concepción sino a la gestación del bebe: para considerarla práctica médica y así legitimarla, hace falta - así como antes hemos notado en casos análogos - pensar en la actividad médica en términos esquisítamente técnicos, justificados por los resultados deseados más que por los resultados terapéuticos. Por este camino, se puede llegar a una legitimación social de la maternidad por surrogación, que muy rápidamente llevaría, como a través de un procedimiento circular, también al reconocimiento clínico (en algunos países de tradición cultural y jurídica anglosajona hace años se ha empezado a proponer, entre el modelo comercial generalmente rechazado y el modelo solidario con sus límites de garantías para con la pareja y el niño, un tercer modelo "profesional"20: aún sin entrar en la discusión acerca de la moralidad de dicha práctica, explican estas autoras sin la mínima sombra de ironía, delante de la objetiva difusión del fenómeno para lograr su pleno control y evitar abusos y explotaciones lo mejor puede resultar profesionalizarla).

Si pasamos a considerar las cuestiones relativas a la parte final de la vida humana,

\footnotetext{
${ }^{17}$ La distinción puede incluso resultar subrepticia y funcional al reconocimiento social y luego legal de la forma oblativa (que supondría aceptación social de la maternidad subrogada): véase en argumento la moción del Comité Nacional de Bioética italiano del mes de marzo de 2016 (<http://presidenza.governo.it/bioetica/mozioni/Mozione_Surroga_materna.pdf>).

${ }^{18}$ Véase el manifiesto online: <http://www.stopsurrogacynow.com/>

${ }^{19}$ Convenio Europeo de Derechos Humanos, art. 8: "(Derecho al respeto a la vida privada y familiar). 1. Toda persona tiene derecho al respeto de su vida privada y familiar, de su domicilio y de su correspondencia. 2. No podrá haber injerencia de la autoridad pública en el ejercicio de este derecho sino en tanto en cuanto esta injerencia esté prevista por la ley y constituya una medida que, en una sociedad democrática, sea necesaria para la seguridad nacional, la seguridad pública, el bienestar económico del país, la defensa del orden y la prevención de las infracciones penales, la protección de la salud o de la moral, o la protección de los derechos y las libertades de los demás" (<http://www.echr.coe.int/Documents/Convention_SPA.pdf>). Alguna reflexión más sobre esos temas en C. Sartea, Aventuras y desventuras del derecho a la privacidad, en VV.AA., Bioética y nuevos derechos, J.A. Santos, M. Albert, C. Hermida (Eds.), Comares, Madrid, 2015, pp. 187 y ss. (desarrolla las aplicaciones del derecho a la privacidad en la fase final de la vida el sucesivo ensayo del mismo volumen: M. Albert, Privacidad y derecho a morir, pp. 203 y ss., con referencias a la jurisprudencia de Estrasburgo).
}

${ }^{20}$ L. Van Zyl, R. Walker, "Beyond altruistic and commercial contract motherhood: the professional model", en Bioethics, 2013 Sep; 27(7):373-81. 
resulta si cabe más fácil aún enterarse de que el argumento jurídico normalmente invocado para pedir la legalización de la eutanasia y del suicidio asistido es el mismo: la autodeterminación del paciente debe ser la principal o única referencia, aceptando incluso el riesgo de la instrumentalización del sanitario; y dicha autodeterminación incluye sin duda estados emotivos y condiciones psicológicas. Hay más: el dolor físico y objetivable como tal no resulta la principal causa de petición eutanásica, según la reciente investigación publicada en el prestigioso "Journal of American Medical Association": "Between $0.3 \%$ to $4.6 \%$ of all deaths are reported as euthanasia or phisician-assisted suicide in jurisdictions where they are legal. The frequency of these deaths increased after legalization. More than $70 \%$ of cases involved patients with cancer. Typical patients are older, white, and well-educated. Pain is mostly not reported as the primary motivation"21.

Incluso la experiencia directa de Holanda confirma que prevalecen entre los criterios operativos los perfiles psicológicos sujetivos respeto a los corporales, que pueden fácilmente quedar objetivados por los clínicos: el "código práctico" de la Comisión Nacional de Eutanasia, en la versión de abril de 2015, explica que "suffering is a broad concept. It can result from pain and shortness of breath, extreme exhaustion and fatigue, but also from growing dependence, feelings of humiliation, physical decline, loss of dignity or the fact that there is no prospect of improvement"; por eso, "there is seldom only one dimension to the burden of suffering experienced by the patient. In practice, it is almost always a combination of aspects, including the absence of any prospect of improvement, which determines whether suffering is unbearable. The physician must therefore investigate all aspects that together make the patient's suffering unbearable"22.

La progresiva sujetivización de los criterios de legitimación - social y luego jurídica - de dichas prácticas, en el marco de las culturas líquidas en que nos encontramos inmergidos, nos habla de un proceso importante de translación de los significados. Resulta cada vez más clara la renuncia al anclaje objetivo de la sabiduría médica - y de la sabiduría jurídica: juris prudentia -, en virtud de la confianza creciente en las capacidades del sujeto interesado (o, que es lo mismo, de la creciente desconfianza hacia las capacidades de los operadores sanitarios, o más en general hacia la posibilidad de encontrar un sólido terreno de enfrentamiento entre las pretensiones del paciente y los "rationals" profesionales). Es muy dudoso que dicha situación pueda mejorar la relación entre médico y paciente: lo que sin duda produce es un aumento incontrolable de la tensión conflictiva subyacente ${ }^{23}$, como por ejemplo resulta bien patente en el citado documento del Comité Nacional de Bioética italiano sobre rechazo y renuncia consciente a los cuidados ${ }^{24}$, que en sus consideraciones finales supone una juridificación de la

${ }^{21}$ E.J. Emanuel et al., Attitudes and Practices of Euthanasia and Physician-Assisted Suicide in the United States, Canada, and Europe, cit., p. 79.

${ }^{22}<$ http://www.euthanasiecommissie.nl/uitspraken/brochures/brochures/code-of-practice/1/code-of-practice>, p. 13

${ }^{23}$ La que ya desde años nos hemos acostumbrado a llamar "medicina defensiva": es decir el conjunto de los intentos de los operatores sanitarios finalizados no ya a la salud del paciente, sino a la propia defensa preventiva en orden a posibles ataques deontológicos o judiciales futuros. El multiplicarse de los procesos de responsabilidad (civil e incluso penal) intentados contra médicos y cirujanos, y las mismas oscilaciones de los jueces de todos los niveles (incluso los de las Cortes Supremas), constituyen señales suficientemente fehacientes del fracaso de todo el esquema relacional.

${ }^{24}<$ http://presidenza.governo.it/bioetica/pareri_abstract/rifiuto_rinuncia_consapevole_paziente_medico_2410 2008.pdf>. Llama la atención el hecho que la parte conclusiva del documento - criticada por algunos miembros del Comité en sus opiniones disencientes en anexo al documento mismo - indique modalidades de solución de las tensiones en una perspectiva esencialmente jurídica, de derechos y deberes contrapuestos: aunque conste el esfuerzo titánico del redactor y de los que aprobaron el documento, para 
DOI: http://dx.doi.org/10.12795/IETSCIENTIA.2016.i02.11

relación clínica presentada como la mejor forma de evitar el riesgo de mutua instrumentalización.

\section{A la búsqueda de un nuevo orden}

Como se ha notado ${ }^{25}$, en todos los casos mencionados nos encontramos por completo dentro de una lógica de derechos sujetivos pensados en términos esencialmente sujetivistas: como espacio de poder que el ordenamiento reconoce al individuo particular, sin que se pueda debatir sobre su legitimación ni sobre su contenido $^{26}$. Pero por esta senda, no es difícil entrever que el futuro es obscuro: como afirma Cotta, "el triunfo de la vida a través de la consagración ética y la legitimación jurídica de la libertad se vierte enseguida en el triunfo inesperado de la muerte: la muerte propia y la muerte ajena" 27 . No es éste, el nihilismo, un éxito aceptable, ya que no resulta compatible ni con las relaciones consideradas (la muerte aún solamente de uno de los contendientes implica el final de toda posibilidad relacional), ni en general con la sobrevivencia del hombre y de su especie.

En analogía a lo que el mismo Cotta sugiere para recuperar el sentido propio del derecho sujetivo, de forma compatible con la juridicidad, podemos aquí proponer una posible salida del impasse. En efectos, solamente encontrando una res intermedia entre dos sujetos, que pueda constituir el objetivo compartido por ambos en sus estrategias operativas, es posible colocarlos en una mutua relación no destructiva sino constructiva: es decir, empezar un diálogo entre ellos. Es lo que, en el debate bioético de hace algunos años, se presentaba como la disyuntiva entre "moral strangers" y "moral friends"28. sin embargo, para llegar a un diálogo no basta con comprobar la distancia (a veces, realmente enorme) entre las posiciones éticas o religiosas de los protagonistas, sino que hay que encontrar un lugar común de contacto $^{29}$. Como afirma D'Agostino, "no existen extranjeros morales: este principio debe quedar asumido como el postulado fundamental de una bioética que sepa ir más allá de la crisis postmoderna. Y se trata de un postulado a la vez teorético, ético y político: teorético, ya que supone la afirmación de la igualdad

respetar de la mejor forma posible las distintas posiciones en juego, resulta claro que la relación médicopaciente sufre un profundo menoscabo por parte de dicho planteamiento.

${ }^{25}$ F. D'Agostino ha hablado, y justamente en relación con cuestiones biojurídicas (II diritto naturale, il diritto positivo e le nuove provocazioni della bioetica, en Id., Parole di bioetica, Giappichelli, Torino, 2006, p. 195), de un inédito "iusnaturalismo libertario": y ha puesto en guardia en orden a una posible intrínseca "peligrosidad" de los derechos fundamentales así planteados (F. D’Agostino, Corso breve di filosofia del diritto, Giappichelli, Torino, 2011, p. 153).

${ }^{26}$ Una crítica a parecido planteamiento está bien presente ya en Sergio Cotta, Attualità e ambiguità dei diritti fondamentali, cit.,p. 24: "Il diritto del soggetto assume un'ampiezza e una fondamentalità senza precedenti. Prima era considerato racchiuso entro una zona ben delimitata della vita, sottratta, perché 'privata', all'imperio della 'pubblica' autoritá, la quale peraltro non era contestata nel suo ambito proprio. Ora ciò che era 'privato' reclama il diritto illimitato di manifestarsi in 'pubblico', di imporsi al 'pubblico', di essere soddisfatto a spese del 'pubblico'”. Véase también M. Villey, Le Droit et les droits de l'homme, PUF, Paris, 1983.

${ }^{27}$ S. Cotta, op. cit., p. 25 (mía la traducción).

${ }^{28}$ La expresión, como es conocido, es de H.T. Engelhardt Jr., Los fundamentos de la bioética, Paidos, Madrid, 1995.

${ }^{29}$ La propuesta biojurídica del libro VV.AA., II filo delle Parche. Opinioni comuni e valori condivisi nel dibattito biogiuridico, F. Zanuso (Ed.), FrancoAngeli, Milano, 2009, ya en el título subraya esta necesidad de buscar y posiblemente encontrar a los "éndoxa", las opiniones compartidas. Una reflexión de Vicente Bellver Capella ("Por un debate constructivo sobre el aborto", en Claves de razón práctica, 233, 2014, pp. 56-65) aboga por esa misma búsqueda en el agrio debate sobre la ley de interrupción voluntaria del embarazo. 
DOI: http://dx.doi.org/10.12795/IETSCIENTIA.2016.i02.11

ontológica entre todos los seres humanos; ético, ya que implica la afirmación axiológica de la fraternidad universal entre todos los hombres; político, porque aparta el debate ético del espacio exclusivo de la conciencia interior y lo coloca en un contexto público"30.

Así las cosas, para que sea posible un diálogo - clínico y jurídico, además que humano y moral - entre paciente y médico, es indispensable que ambos acepten un horizonte de referencia común, que es el mismo que justifica la necesidad del paciente (causa de su dirigirse al médico, es decir, origen primero, existencial y fáctico, de la relación) y el involucramiento del médico (escogido por sus competencias y habilidades específicas, como profesional del sector). Ese punto de contacto del que depende la misma posibilidad del diálogo no puede ser sino la salud de la persona humana, pero una salud necesariamente pensada de forma objetiva y no solamente subjetiva: aunque en su mismo concepto haya que incluir también los elementos de bienestar psicológico y espiritual del paciente y de su entorno. De otra forma, el abandono de la objetividad implicaría la pérdida del sentido mismo de la profesionalidad y la corrupción insanable de la relación clínica. No pienso dramatizar el asunto, desde luego: sencillamente, la relación entre paciente y médico pasa de ser un pacto finalizado a la salud (según los casos, to cure o bien to care), a otros significados, que por eso mismo quedan consignados a la libre interpretación de las partes involucradas y sólo por casualidad llegarán a un lugar común de armonización. En este diferente contexto, ni la filosofía de la medicina ni la filosofía del derecho y la biojurídica podrán hacer mucho para prevenir los conflictos.

\section{Una conclusión}

Como otras veces ha pasado en épocas anteriores, el interesante paralelismo entre clínica y derecho vuelve a asomarse y tal vez nos muestra el sendero. Si la medicina, en su identidad propia (la única que puede justificar su actuación social, su reconocimiento público, su prestigio cuando lo hay, etc.), es cura del sujeto, y no de la sujetividad (entendiendo esta última como el conjunto de las manifestaciones de una voluntad particular), de la misma manera podemos afirmar que el derecho es protección del sujeto, y no de su sujetividad. La medicina trata de la vida y de la salud del paciente objetivamente interpretadas, y el derecho protege y promueve los derechos del sujeto considerados en cuanto bienes objetivos, que todo sujeto merece ver protegidos en favor de su coexistencia con otros seres humanos. Es conocido que hay muchas teorías distintas, y a veces incompatibles, sobre los bienes humanos que objetivamente merecen ser amparados por el derecho: ahora no nos interesa referirnos a este importante debate en torno al contenido de las muchas listas que los filósofos del derecho han propuesto (una de las últimas y más famosas es la de Finnis ${ }^{31}$ ), sino notar que todas estas listas, en su esencia, llegan a considerar que existe un patrimonio común de los seres humanos que constituye como el cogollo de su dignidad y la premisa de su posibilidad de vivir en sociedad.

Para concluir, entonces, nos basta con recordar que según Cotta es la coexistencia el bien mínimo básico del que depende todo reconocimiento de derechos fundamentales (o humanos, que en este orden de discurso coinciden). "La coexistencia es necesidad ontológica fundamental, por tanto el principium reddendae rationis, es decir el fundamento, de ser-ahí de los diversos modos de relación interhumana (de amistad, económica, política, jurídica, moral). Pero es también el criterio fundamental de valor de su constitución y desarrollo, porque permite juzgar objetivamente (es decir con una

\footnotetext{
${ }^{30}$ F. D’Agostino, Bioetica nella prospettiva della filosofia del diritto, cit., p. 11 (mía la traducción).

${ }^{31} \mathrm{~J}$. Finnis, Ley natural y derechos naturales, (1980), trad. C. Orrego, Abeledo-Perrot, Buenos Aires, 2000.
} 
validez comprobable universalmente) su correspondencia, más o menos amplia y más o menos profunda, con la exigencia de llevar a plenitud el ser del existente a lo largo de su actuación para acogerse mutuamente, satisfaciendo así la necesidad ontológica de coexistencia"32.

Puede parecer poca cosa: y sin embargo, hace que sea posible todo lo demás.

\section{Bibliografía citada}

Bauman, Z. Vida líquida, (2005), trad. A. Santos, Paidos, Barcelona, 2006.

Bauman, Z.Amor líquido. Acerca de la fragilidad de los vínculos humanos, (2005), trad. M. Rosenberg y J. Arrambide, FCE, Madrid, 2006.

Bellver Capella, V. "Por un debate constructivo sobre el aborto", en Claves de razón práctica, 233, 2014.

Cotta, S. "La coexistencialidad como fundamento ontológico del derecho", en Persona y Derecho, 1982, IX.

Cotta, S. Attualitá e ambiguitá dei diritti fondamentali, in Id., I/ diritto come sistema di valori, San Paolo, Milano, 2004.

D’Agostino, F.Bioetica nella prospettiva della filosofia del diritto, Giappichelli, Torino, 1996.

D’Agostino, F.Parole di bioetica, Giappichelli, Torino, 2006.

D’Agostino, F. Introduzione ai lavori: paranoie della modernitá, en VV.AA., Autodeterminazione: un diritto di spessore costituzionale?, Giuffrè, Milano, 2010.

D’Agostino, F. Corso breve di filosofia del diritto, Giappichelli, Torino, 2011.

Emanuel E.J. et alii.Attitudes and Practices of Euthanasia and Physician-Assisted Suicide in the United States, Canada, and Europe, JAMA, 2016; 316(1): 79-90.

Engelhardt Jr., H.T. Los fundamentos de la bioética, Paidos, Madrid, 1995.

Finnis, J. Ley natural y derechos naturales, (1980), trad. C. Orrego, Abeledo-Perrot, Buenos Aires, 2000.

Sartea, C. Deontologia. Filosofia del lavoro professionale, Giappichelli, Torino, 2010.

Sartea, C. "Bioetica giudiziaria in Italia: note critiche su una sentenza recente in tema di protezione della vita prenatale", en Ius Humani. Revista de Derecho, Vol. 4 (2014/2015).

Spagnolo, A.G. "Il suicidio assistito dell'autonomia", en Medicina e Morale, 2015/4.

Van Zyl, L., Walker, R. "Beyond altruistic and commercial contract motherhood: the professional model", en Bioethics, 2013 Sep; 27(7):373-81.

Villey, M. Le Droit et les droits de l'homme, PUF, Paris, 2014.

VV.AA. Questioni di bioetica, a cura diS. Rodotá, Laterza, Roma-Bari, 1997.

VV.AA.Filosofia della medicina, a cura di G. Federspil, P. Giaretta, C. Rugarli, C. Scandellari, P. Serra, Cortina, Milano, 2008.

VV.AA., Il filo delle Parche. Opinioni comuni e valori condivisi nel dibattito biogiuridico, F.

\footnotetext{
${ }^{32}$ S. Cotta, "La coexistencialidad como fundamento ontológico del derecho", en Persona y Derecho, 1982, IX, p. 18.
} 
Zanuso (Ed.), FrancoAngeli, Milano, 2009.

VV.AA. Diritto e desiderio. Riflessioni biugiuridiche, F. Zanuso (ed.), Milano, FrancoAngeli, 2015.

VV.AA., Bioética y nuevos derechos, J.A. Santos, M. Albert, C. Hermida (Eds.), Comares, Madrid, 2015. 